\title{
Long-Term Outcomes of Acute Low-Tone Hearing Loss
}

\author{
Kyung Jin Roh, Eun Jung Lee, Ah Young Park, Byeong II Choi, and Eun Jin Son \\ Department of Otorhinolaryngology, Yonsei University College of Medicine, Seoul, Korea
}

\section{Received December 16, 2014 \\ Revised March 16, 2015 \\ Accepted May 11, 2015}

Background and Objectives: Although acute low-tone hearing loss has been associated with cochlear hydrops or early stage Meniere's disease, its prognosis in the short-term has been reported to be better than sudden hearing loss. However, recurrence of hearing loss and possible progression to Meniere's disease remain important concerns in the clinical setting. This study aims to investigate the long-term audiological outcomes of acute low-tone hearing loss. Subjects and Methods: A retrospective review of patients presenting with a first attack of acute low-tone hearing loss was performed. Of the 77 patients, 33 were followed up for more than 3 months. Progression, recovery of hearing loss and recurrence of hearing loss were examined. Also, correlation between long-term outcomes and associated clinical factors were analyzed. Results: Twenty-five patients (75.7\%) had complete hearing recovery, five patients (15.1\%) had partial recovery, two patients $(6.0 \%)$ had no recovery, and one patient (3.0\%) had progression of hearing loss 1 month after initial treatment. Thirty-three patients were followed up for more than 3 months (mean 22 months, range 3-79 months). Recurrences of acute low-tone hearing loss were observed in five patients (15.2\%). All of the recurrences occurred during the first 12 months of follow-up. Long-term prognosis correlated with the initial therapy results $\left(R^{2}=0.693\right)$. Conclusions: Recurrences of hearing loss were documented in five patients (15.2\%), and all of these cases occurred within one year of the first attack. Audiological outcomes after initial therapy may predict the recurrence of acute low-tone hearing loss.

J Audiol Otol 2015;19(2):74-78

\section{Introduction}

Acute low-tone sensorineural hearing loss (ALHL) was initially considered as a variant of idiopathic sudden sensorineural hearing loss [1] until its first description as an independent disease entity by Abe in 1982 [2]. Although the audiometric definition of ALHL is not yet fully established, one of the most widely applied diagnostic criteria was proposed by the Study Group for Acute Profound Deafness Research Committee of the Ministry of Health, Labor, and Welfare of Japan. It defines ALHL as acute onset of hearing loss with the sum of hearing levels in affected ears at low frequencies of 125,250 , and $500 \mathrm{~Hz}$ as $70 \mathrm{~dB}$ or more, and the sum of hearing levels at high frequencies of 2,4 , and $8 \mathrm{kHz}$

This is an Open Access article distributed under the terms of the Creative Commons Attribution Non-Commercial License (http://creativecommons. org/licenses/by-nc/3.0/) which permits unrestricted non-commercia use, distribution, and reproduction in any medium, provided the original work is properly cited. as $60 \mathrm{~dB}$ or less. The pathophysiology of ALHL is yet unknown but is suggested to be similar to idiopathic sudden deafness [3]. It has been associated with cochlear hydrops or early stage Meniere's disease [3-5]. Steroids or mild diuretics have been used for clinical treatment, and the prognosis in the short-term has been reported to be better than sudden hearing loss [2]. However, recurrence of hearing loss and possible progression to Meniere's disease remain important concerns in the clinical setting $[4,6,7]$. In a previous report, the rate of recurrence was about $40 \%$ [8], and the rate of progression to Meniere's disease was about 11\% [4].

This study aims to examine the clinical course of ALHL patients during long-term follow-up of more than 3 months and to identify associated factors.

\section{Subjects and Methods}

We retrospectively reviewed the medical records of 105 pa- 
tients presenting with a first attack of acute low-tone hearing loss in the Department of Otorhinolaryngology of Yonsei University Gangnam Severance Hospital from January 2007 to January 2013. ALHL was defined by the following criteria: an acute onset sensorineural hearing loss at low frequencies $(250$ and $500 \mathrm{~Hz})$; a sum of hearing levels at low frequencies $\geq 50 \mathrm{~dB}$; and a hearing threshold difference between both ears $<10 \mathrm{~dB}$ at high frequencies ( 2 and $4 \mathrm{kHz}$ ). Patients who presented after more than 2 weeks from onset of symptoms were excluded. Patients with the following criteria were also excluded: 1) evidence of sound conduction deficit; 2) bilateral involvement; 3 ) history of episodic vertigo or presence of nystagmus at initial visit; and 4) neurological abnormalities on history review or physical examination. The remaining 77 patients were included in our study. Of the 77 patients, 33 were followed up for more than 3 months. The patients were routinely examined by pure tone average. Treatment modalities included systemic oral corticosteroids and/or diuretics and/or intratympanic dexamethasone injection. Corticosteroid treatment consisted of 30-60 mg methylprednisolone per day as a starting dose administered orally and tapering for 10 days. Diuretic treatment consisted of a daily oral $50 \mathrm{mg}$ spironolactone for 2 weeks. Intratympanic injections of 0.5 cc dexamethasone $(5 \mathrm{mg} / \mathrm{mL})$ for 5 days were performed. At 1 month after the first visit, the results of initial therapy were evaluated according to the following criteria: complete recovery if hearing thresholds at low frequencies were less than $20 \mathrm{~dB}$ or the same level as the opposite ear; partial recovery if the average of hearing thresholds at low frequencies improved more than $10 \mathrm{~dB}$ but was not at the same level as the opposite ear; unchanged (UC) if the average hearing thresholds at low frequencies improved $<10 \mathrm{~dB}$; and progressive changes for patients with hearing changes other than those in the aforementioned three categories [9]. Hearing evaluations were performed at regular intervals: at initial visit, after one month, then at 3- or 6-month intervals. The progression or recovery of hearing loss and recurrence of hearing loss were examined, and correlation between long-term outcomes and associated clinical factors were analyzed.

\section{Statistical analysis}

All statistics were calculated with SPSS v20.0 for Windows (SPSS Inc., Chicago, IL, USA). $p$-value $<0.05$ was considered statistically significant. Statistical evaluation regarding correlation between various intervals was performed using bivariate correlation analysis. The analysis of recurrence-free rate was performed using Kaplan-Meier test.

\section{Results}

Table 1 summarizes the clinical characteristics of 33 patients with ALHL who were followed up for more than 3 months. M:F ratio was 13:20, and mean age was 41 years (range 14-69 years). Hearing loss was in the left ear in 20 patients and the right ear in 13. Mean follow-up period was 22 months (range 3-79 months).

Hearing thresholds were measured at initial presentation (Fig. 1). The hearing thresholds (mean $\pm \mathrm{SD}$ ) in the affected ear at each frequency were: $49 \pm 43 \mathrm{~dB} \mathrm{HL}$ at $250 \mathrm{~Hz} ; 45 \pm$ $17 \mathrm{~dB} \mathrm{HL}$ at $500 \mathrm{~Hz} ; 33 \pm 21 \mathrm{~dB} \mathrm{HL}$ at $1 \mathrm{kHz} ; 23 \pm 16 \mathrm{~dB}$ $\mathrm{HL}$ at $2 \mathrm{kHz} ; 22 \pm 14 \mathrm{~dB} \mathrm{HL}$ at $4 \mathrm{kHz}$; and $27 \pm 17 \mathrm{~dB} \mathrm{HL}$ at $8 \mathrm{kHz}$. In the opposite ear, the hearing thresholds (mean \pm $\mathrm{SD})$ at each frequency were: $11 \pm 7 \mathrm{~dB} \mathrm{HL}$ at $250 \mathrm{~Hz} ; 10 \pm 7$ $\mathrm{dB} \mathrm{HL}$ at $500 \mathrm{~Hz} ; 12 \pm 8 \mathrm{~dB} \mathrm{HL}$ at $1 \mathrm{kHz} ; 17 \pm 22 \mathrm{~dB} \mathrm{HL}$ at

Table 1. Summary of clinical characteristics

\begin{tabular}{lc}
\hline & Total $(\mathrm{n}=33)$ \\
\hline Age & \\
Avg \pm SD (yrs) & $41 \pm 12.68$ \\
Range (yrs) & $14-69$ \\
Gender (M:F) & $13: 20$ \\
Laterality (R:L) & $13: 20$ \\
F/u period & \\
Avg $\pm S D$ (months) & $22 \pm 21.23$ \\
Range (months) & $3-79$ \\
Recurrence & $5(15.2 \%)$ \\
\hline
\end{tabular}

M: male, F: female, R: right, L: left, SD: standard deviation, F/U: followed up, Avg: average, yrs: years

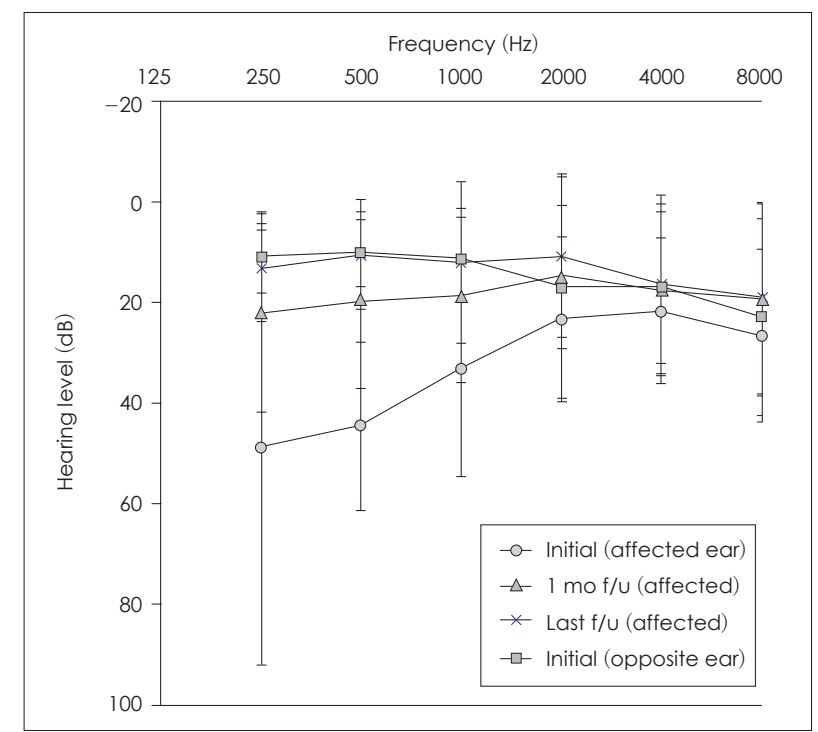

Fig. 1. Pure tone average at various intervals. Median air conduction thresholds in both ears (affected and opposite) at initial presentation, in affected ears at 1 month, and in affected ears at the last f/u visit. f/u: followed up. 
$2 \mathrm{kHz} ; 17 \pm 15 \mathrm{~dB}$ HL at $4 \mathrm{kHz}$; and $23 \pm 20 \mathrm{~dB} \mathrm{HL}$ at $8 \mathrm{kHz}$. Affected ear audiogram at initial visit shows an ascending typed graph, and at high frequencies, the difference in threshold between the affected ear and the opposite ear was less than $10 \mathrm{~dB}$. According to audiograms after 1 month and at last examination, the recovery of hearing loss at low frequencies occurred mostly in the first month.

After initial treatment at 1 month, 25 patients $(75.7 \%)$ had complete hearing recovery, five patients $(15.1 \%)$ had partial recovery, two patients $(6.0 \%)$ had no recovery, and one patient (3.0\%) had progression of hearing loss (Fig. 2). The results of hearing level at the last follow-up showed a few improvements compared to 1 month after initial visit, but the changes were not statistically significant. At the last followup, 29 patients $(87.8 \%$ ) had complete hearing recovery, three patients $(9.0 \%)$ had partial recovery, and one patient $(3.0 \%)$ had no recovery. We calculated the decibel-sum (dB-sum) of hearing thresholds at 250 and $500 \mathrm{~Hz}$ to analyze the correla-

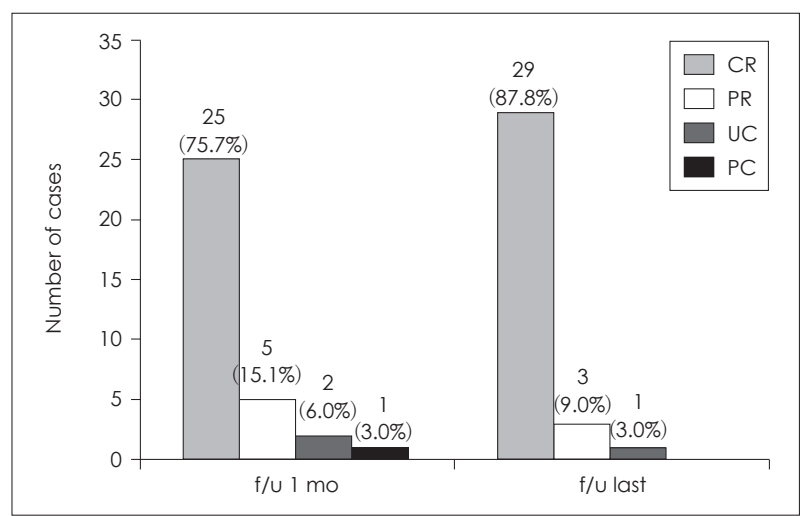

Fig. 2. Analysis of recovery outcomes. After initial treatment at 1 month, 25 patients $(75.7 \%)$ had complete hearing recovery, five patients $(15.1 \%)$ had PR, two patients $(6.0 \%)$ had no recovery, and one patient $(3.0 \%)$ had progression of hearing loss. At the last f/u, 29 patients $(87.8 \%)$ had complete hearing recovery, three patients $(9.0 \%)$ had PR, and one patient $(3.0 \%)$ had no recovery. CR: complete recovery, PR: partial recovery, UC: unchanged, PC: progressive change, f/u: follow-up. tion between audiological outcomes at various intervals (Fig. 3). Although there was no significant correlation between the $\mathrm{dB}$-sum of the affected ears at initial presentation and last follow-up (Fig. 3A), a positive linear correlation was observed between the $\mathrm{dB}$-sum at 1 month and last follow-up (Fig. 3B) $\left(\mathrm{R}^{2}=0.693\right)$. The results suggest that the long-term audiological outcomes show better correlation with the hearing thresholds at 1 month after initial treatment than with the initial hearing levels. Recurrence of acute low-tone hearing loss was observed in five patients $(15.2 \%)$. All of the recurrences occurred during the first year of follow-up. Three patients presented with recurred hearing loss within 3 months, and two patients between 3 months and one year (Fig. 4).

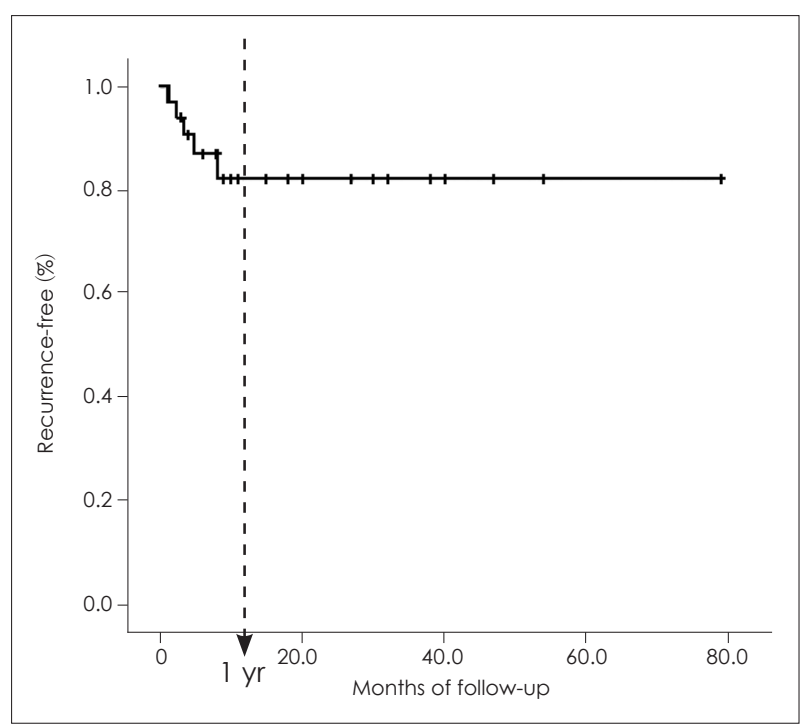

Fig. 4. Kaplan-Meier plots of 33 patients with ALHL indicating the rate and timing of recurrence of symptoms during follow-up. Recurrence of ALHL was observed in five patients (15.2\%). All of the patients with recurrence experienced it within 1 year of the first episode of hearing loss. ALHL: acute low-tone sensorineural hearing loss, yr: year.

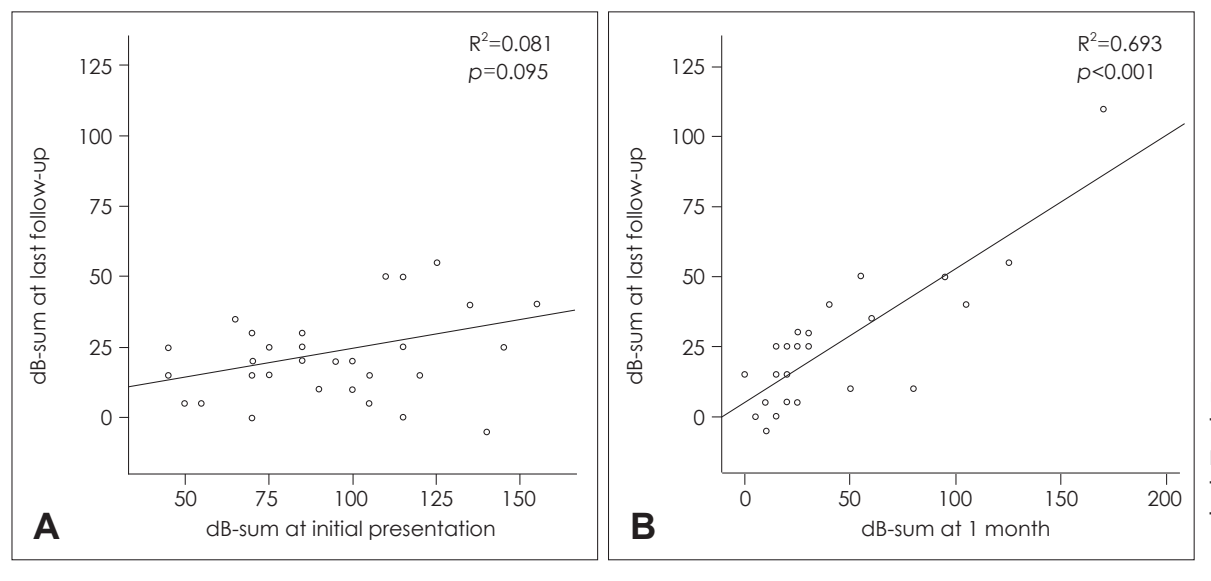

Fig. 3. Low-tone dB-sum of hearing thresholds at 250 and $500 \mathrm{~Hz}$ correlation analysis. A: Correlation between initial presentation and last follow-up. B: Correlation between 1 -month visit and last follow-up. 


\section{Discussion}

ALHL is known to have a relatively good prognosis and generally improves within a short time after treatment [10]. However, fluctuation of hearing loss or progression to overt Meniere's disease has been reported [6]. Few reports are available about the long-term outcomes of ALHL. Oishi, et al. [11] reported that about half of the cases in their study developed high- or pan- frequency hearing loss within 10 years of onset of low-frequency hearing loss. They also reported that the initial therapy results and fluctuation of hearing during the first year may indicate the long-term prognosis of patients presenting with low-frequency hearing loss. In our study, the audiological outcomes of ALHL patients were examined for more than 3 months. Our findings indicate that in most patients, the hearing levels were improved within 1 month after initial treatment with steroids and/or diuretics, in accordance with other studies. A previous report by Oishi, et al. [11] indicated that hearing fluctuation during the first year after the initial attack correlated with the 10-year prognosis of ALHL ( $r=0.43$ ). Considering that recurrence of hearing loss was documented in five patients $(15.2 \%)$ within our one-year period, longer follow-up of these cases would be needed.

Predictive factors of ALHL have been investigated. Yamasoba, et al. [4] reported that ratios of the summating potential to the action potential (SP/AP ratios) were abnormally increased in electrocochleography (ECoG) in most patients with ALHL. In the study of Fushiki, et al. [8], patients with an elevated SP/AP ratio and spontaneous nystagmus on electronystagmography had a recurrence rate of approximately $80 \%$ after long-term follow-up. The study demonstrated that a combination of nystagmus detection and ECoG testing could improve the accuracy of both prognosis and treatment planning in patients with sudden low-tone sensorineural hearing loss without vertigo [8]. These findings support the possibility that ALHL overlaps with an early stage of Meniere's disease or endolymphatic hydrops of the cochlea. In addition, Hong, et al. [12] reported that cochlear hydrops analysis masking procedure (CHAMP) measurement may be useful for determining the prognosis of patients with ALHL without vertigo. They observed that patients with an abnormal complex amplitude ratio on CHAMP had a 2.6-fold increased risk of progression to a clinical spectrum of endolymphatic hydrops. Although we could not determine the correlations between various audiovestibular examinations and hearing outcomes due to a relatively small number of cases, our findings suggest that hearing levels after initial treatment may predict the long-term audiological outcomes in ALHL.

One of the limitations of our study is that only 33 of total 77 patients were followed up for more than 3 months. When we included the patients who were lost to follow-up in the initial treatment outcome analysis, UC group increased from 6 to $16 \%$. The patients were likely unsatisfied with the initial treatment outcome and showed higher dropout rate. Our results should be interpreted with caution to this selection bias, and further study about ALHL patients need to be followed through a prospective study with fewer dropouts.

To our knowledge, there is no report that correlates the prognosis of ALHL with severity of initial hearing loss. In our study, hearing recovery was not correlated with severity at initial presentation, but with the initial treatment outcome at 1 month. Since, most of ALHL patients present with mild to moderate hearing loss restricted in the low frequencies, further studies of larger groups of patients are warranted to investigate the correlation.

\section{Conclusion}

Our results suggest that the hearing levels after initial treatment correlate with long-term outcomes in ALHL. Also, recurrence of hearing loss generally occurred during the first year after initial presentation, suggesting the importance of longer follow-up for ALHL patients.

\section{Acknowledgments}

This work is supported by a faculty research grant of Yonsei University College of Medicine (6-2012-0023) to E.J.S.

\section{REFERENCES}

1) Shaia FT, Sheehy JL. Sudden sensori-neural hearing impairment: a report of 1,220 cases. Laryngoscope 1976;86:389-98.

2) Abe T. Acute sensorineural hearing loss in low frequencies. Otolaryngol Tokyo 1982;54:8.

3) Yamasoba T, Sugasawa M, Kikuchi S, Yagi M, Harada T. An electrocochleographic study of acute low-tone sensorineural hearing loss. Eur Arch Otorhinolaryngol 1993;250:418-22.

4) Yamasoba T, Kikuchi S, Sugasawa M, Yagi M, Harada T. Acute lowtone sensorineural hearing loss without vertigo. Arch Otolaryngol Head Neck Surg 1994;120:532-5.

5) Noguchi Y, Nishida H, Tokano H, Kawashima Y, Kitamura K. Comparison of acute low-tone sensorineural hearing loss versus Meniere's disease by electrocochleography. Ann Otol Rhinol Laryngol 2004;113 (3 Pt 1):194-9.

6) Imamura S, Nozawa I, Imamura M, Murakami Y. Clinical observations on acute low-tone sensorineural hearing loss. Survey and analysis of 137 patients. Ann Otol Rhinol Laryngol 1997;106:746-50.

7) Abe T, Kon Y, Murai K, Tsuiki T. [Clinical pictures of low tone sudden deafness]. Nihon Jibiinkoka Gakkai Kaiho 1988;91:667-76.

8) Fushiki H, Junicho M, Aso S, Watanabe Y. Recurrence rate of idiopathic sudden low-tone sensorineural hearing loss without vertigo: a long-term follow-up study. Otol Neurotol 2009;30:295-8.

9) Kawashima Y, Satoh H, Okamoto M, Nakashima T, Ihara K, Kitamura K. Epidemiological study of acute low-tone sensorineural hearing loss in Kanagawa and Iwate Prefectures. Audiol Jpn 2006;49: $373-80$ 
10) Fuse T, Aoyagi M, Funakubo T, Sakakibara A, Yoshida S. Short-term outcome and prognosis of acute low-tone sensorineural hearing loss by administration of steroid. ORL J Otorhinolaryngol Relat Spec 2002;64:6-10.

11) Oishi N, Inoue Y, Saito H, Kanzaki S, Kanzaki J, Ogawa K. Longterm prognosis of low-frequency hearing loss and predictive factors for the 10-year outcome. Otolaryngol Head Neck Surg 2010;142: 565-9.

12) Hong SK, Nam SW, Lee HJ, Koo JW, Kim DH, Kim DR, et al. Clinical observation on acute low-frequency hearing loss without vertigo: the role of cochlear hydrops analysis masking procedure as initial prognostic parameter. Ear Hear 2013;34:229-35. 